\section{Mental health during COVID-19 in Qatar}

\author{
Ovais Wadoo (D) ,' Javed Latoo, ${ }^{1}$ Shuja Mohd Reagu, ${ }^{1}$ \\ Raed Ahmad Assi Amro, ${ }^{1}$ Naseer Ahmad Masoodi, ${ }^{2}$ \\ Majid Alabdulla ${ }^{1,3}$
}

\section{INTRODUCTION}

The COVID-19 pandemic has posed unprecedented health and social challenges. WHO has been working to promote the integration of mental health and psychosocial support into the COVID-19 response effort. ${ }^{1}$ It is vital that targeted measures are initiated to mitigate the potential mental health consequences of the COVID-19 pandemic and psychiatric patients continue to receive a range of psychiatric services. Countries need to adapt strategies identified by WHO to strengthen mental healthcare delivery.

\section{MENTAL HEALTH SERVICES IN THE STATE OF QATAR}

Qatar is a peninsula located amid the western coast of the Arabian Gulf. It occupies an area of $11521 \mathrm{~km}^{2}$. It has a population of 2.7 million. ${ }^{2}$ Of the total population, $89 \%$ are expatriates from over 150 countries. About $60 \%$ of the population consists of craft and manual workers (CMWs), typically working in mega-development projects. It has the highest income per capita in the world due to its substantial reserves of oil and gas. It represents a unique healthcare system due to its rapid economic growth and the population it serves. Mental health, in recent years, has been included as a priority in the national health strategy, ${ }^{3}$ and this has led to improvements in facilities, recruitments and services. Qatar's mental health service is still in an early phase of development, and there are plans to increase

\footnotetext{
${ }^{1}$ Department of Psychiatry, Mental Health Services, Doha, Qatar

${ }^{2}$ Ambulatory General Internal Medicine, Hamad Medical Corporation, Doha, Qatar

${ }^{3}$ Department of Psychiatry, Qatar University College of Medicine, Doha, Ad Dawhah, Qatar

Correspondence to Dr Ovais Wadoo; owadoo@gmail.com
}

both the inpatient capacity and community mental health services. ${ }^{4}$

\section{CHALLENGES TO MENTAL HEALTH SERVICES DUE TO COVID-19}

Extent of COVID-19 pandemic in Qatar

Qatar is one of the countries that have the highest numbers of COVID-19positive patients per million population. ${ }^{5}$ By July 11, 103128 SARS-CoV-2 infections had been laboratoryconfirmed at a rate of 36729 per million population. Epidemic intensity in Qatar reflected the unique demographic and residential dwelling structure of this country. The most affected subpopulation was that of the single CMWs living in shared housing accommodations, who work together during the day and typically live together in large dormitories where they share rooms, bathrooms and cafeteria-style meals. In these settings, the options for effective social and physical distancing are limited. ${ }^{6}$

\section{Mental health consequences of COVID-19}

Global COVID-19 pandemic research is indicative of adverse impact on the mental health of people with pre-existing mental health disorders and previously healthy people as well. ${ }^{78}$ This is attributed to neuropsychiatric manifestations of people directly affected by SARS-CoV-2 and indirectly through associated lockdown, containment strategies and the resulting economic slowdown. This has led to an increase in demand for mental health services. In Qatar, this is reflected by more than 10000 calls to mental health helplines from March to July 2020. This is bound to increase further as the pandemic unfolds.

Curtailment of community and ambulatory services

Community and ambulatory mental health services are greatly curtailed in this pandemic by large-scale lockdown and home confinement. Quarantine and isolation measures have been implemented to limit the spread of infection. The Ministry of Public Health $(\mathrm{MoPH})$ in Qatar advised minimising direct contact with patients for non-urgent care. These restrictions had a huge impact on the provision of psychiatric services. All routine outpatient clinics, daycare services and community outreach services were suspended as part of the containment strategy.

\section{Challenges to provision of inpatient services}

Mental health inpatient services are not geared to manage physical health in general and infectious diseases in particular. Infection control in psychiatric units faces unique challenges due to the characteristics of the patients and facilities. ${ }^{9}{ }^{10}$ Comorbidities, such as diabetes, hypertension and obesity, have a greater impact on patients with mental illness. They are more vulnerable to be infected as their cognitive and behavioural symptoms can hamper compliance with confinement and hygiene guidelines. ${ }^{11}$ This necessitated a change in the way we provide services to minimise the risk of infection in patients who are admitted to psychiatric units and extend the services to support people managed in general hospitals.

\section{Cultural challenges}

Qatar is an Arab Muslim country where religious and social norms shape the presentation of psychological distress and help-seeking behaviours. Despite their high prevalence, public knowledge about mental disorders is poor . Negative perceptions ${ }^{12}$ and significant stigma are associated with mental disorders. ${ }^{13}$ Behavioural abnormality are attributed to supernatural phenomena. The public are reluctant to engage in mental health services, and faith healers are generally the first resort for help for any behavioural disorders. Extended families normally live together, with stronger family bonds than that in Western countries. Collective 
interests generally outweigh the individual interests.

\section{Limited high-quality data on mental health consequences of COVID-19}

Research output from the Arabian Gulf region is generally low owing to poor existing research infrastructure and limited experts. There is limited data at present on the prevalence of psychological distress caused by COVID-19 in the Arabian Gulf region, including Qatar. The prevalence of mental disorders in Qatar is comparable with the international data. Anxiety disorder is the most common mental disorder in Qatar ${ }^{14}$, while depression is the most common cause of disability in Qatar, only second to heart disease.

\section{MENTAL HEALTH SERVICES RESPONSE TO COVID-19 IN QATAR}

COVID-19 has required us to quickly adjust the way we work and how we deliver care to our patients. We have been required to find ways that minimise the need for patients to attend our facilities, in order to protect both patients and our staff while providing essential mental health services. These enormous challenges in a developing healthcare system like Qatar necessitated pandemic-mitigation strategies to meet the needs of the most vulnerable in the society. Over the course of the epidemic, MoPH prioritised public mental healthcare and supported service reorganisation and development of new services through the National Pandemic Preparedness Committee. The mental health service rapidly initiated new services and developed procedures and guidelines to address these challenges.

\section{Changes to inpatient hospital and acute}

care

Qatar's only psychiatric inpatient hospital that receives acute admissions from all over the country was designated as a non-COVID-19 site. It necessitated an effective mental health triage strategy. In almost all cases, admissions were directed through the emergency department, limiting direct admissions from the community or criminal justice system. This was to ensure that all potential admissions were screened for infection and tested before admission if deemed high risk. The policy of using appropriate personal protective equipment (PPE) for suspected, unknown and positive cases, while being assessed for admission, was developed and implemented. Patients who were low risk for COVID-19 infection were admitted to the main psychiatric facility. Patients who tested positive or deemed high risk for infection but needed psychiatric inpatient care were directed to a limited number of specialist psychiatry beds set up elsewhere.

Strategy to minimise spread of infection in the main psychiatry hospital

Strategies to minimise spread of infection at the main facility were based around the patient journey through the hospital services so as to manage risk of entry and transmission of the infection at every step of the journey. Infection screening tools based on available evidence were implemented by the mental health services. A single point of entry was designated for the whole facility. Physical barriers were put in place to guide the incoming patients and visitors towards infectionscreening facilities, which were manned by trained nursing and security staff. Extra stations dispensing alcohol gel with hand hygiene instructions were set up. Information technology systems were installed to allow the whole multidisciplinary team to participate in the ward round for patients remotely without being physically in the same room. Hand gel, face masks and full PPE were made available on the inpatient units to be used according to policies and guidelines. Spaces were ensured on each ward to nurse patients who developed possible symptoms while waiting to get tested for the infection. Patient visiting frequency and duration were adjusted and kept to a bare minimum. Visitors were also screened. The movement of the patients in and out of the inpatient units was also limited to the minimum, and infection control measures were observed.
The allocated nurses would discuss infection control measures with each patient at the earliest opportunity. Group activities were kept to small groups and carried out in larger spaces where physical distancing could be maintained. Healthcare workers who could work remotely were advised to do so. Healthcare workers who had to be present were given infection control support by COVID-19 infection control nurses. Healthcare workers were given training and guidance on self-screening and advised not to attend if symptomatic. Additional screening using thermometer and a phone screening application was done. Posters on infection control measures were posted in all units. As the pandemic progressed, masks were made mandatory before entering the hospital buildings.

Similar recommendations have been made by D'Agostino and colleagues ${ }^{15}$ for inpatient wards.

\section{Psychiatric beds in COVID-19 designated} hospitals

Patients who tested positive or deemed high risk for infection but needed psychiatric inpatient care were directed to a limited number of specialist psychiatry beds set up in COVID-19-designated hospitals. This allowed for joint management of such patients by psychiatric teams cooperating with medical teams. Psychiatric beds have now become an integrated part of these designated facilities. To provide safe and effective psychiatric care at these COVID19-designated facilities, mental health nurses, psychiatric trainees and consultant psychiatrists were redeployed from the main psychiatric hospitals to COVID-19 hospitals. The cooperation between psychiatric and medical teams helped to prevent the outbreak of the COVID-19 infection in non-COVID-19 facilities, including the main psychiatric hospitals. Clinical pathways were updated to streamline the provision of psychiatric care to COVID-19-positive patients throughout their hospital journey, including their admission, hospital stay, transfer to step down COVID-19 treatment sites and follow-up after the 
discharge. Resource allocation has been a challenge due to the scarcity of trained mental healthcare workers. Redeployment of healthcare workers to areas in urgent need has been common.

\section{Consultation-liaison services in general} hospitals

All hospitals have consultationliaison services integrated. The new COVID-19 hospitals opened during this pandemic were linked with the existing liaison services. A hybrid model of working was implemented: working remotely when possible and reserving face-to-face contact for circumstances where benefits outweighed the risks. The adaptation was necessary to prevent spread of infection and preservation of workforce.

\section{Modifications to ambulatory and community care}

Community and ambulatory services were greatly curtailed in this crisis by large-scale home confinement. This was circumvented by equipping outpatient departments with systems that allowed for telepsychiatry consultations using both phone and video calls. This was coupled with a new initiative wherein pharmacy teams dispatched medication orders to patients using QPost (national postal service). Most of the medications were delivered by QPost to patients' home, thereby reducing visits to pharmacies and healthcare facilities. Patients with severe mental disorders were stratified and prioritised based on their clinical condition, risks and need for close monitoring. Community outreach interventions were only provided for a limited number of patients, and a telephone screening was carried out prior to home visits. Changes in policies as recommended by Siskind and colleagues ${ }^{16}$ were adapted to allow for more flexible monitoring for clozapine and lithium to minimise risk of infection. Daycare facilities were suspended.

\section{National mental health helpline}

This initiative was introduced during this pandemic due to an anticipated need for mental health support for the population of Qatar. This helpline provides support for people experiencing psychological distress, including the young and elderly, the quarantined, those with pre-existing mental health conditions and frontline healthcare professionals. This new virtual service started in March 2020 and enabled the general public to seek timely help from mental health professionals. This is a unique tiered helpline that is the first of its kind in the region combining helpline and crisis services. People have access to tiered services based on their needs. A wide range of services are offered, including interventions from basic psychological support, structured psychological intervention or assessment to intervention from psychiatrists. All calls are received by the tier 1 triage team who conduct an initial assessment and offer basic psychological aid. Calls that require structured psychological interventions are passed on to the tier 2 team, which is comprised of clinical psychologists. Complicated calls that may require assessment or intervention from a psychiatrist are forwarded to a tier 3 team. This service, being culturally more acceptable, has been well accepted as people with psychological issues are usually reluctant to seek help from mental health services in this region. The service offers a single point of contact for any psychosocial needs and has received more than 10000 calls so far.

\section{Psychological support for people in isolation and quarantine centres}

In the last week of February 2020, a task force was created under the directions of $\mathrm{MoPH}$ to manage quarantine sites operations, to respond to the COVID-19 pandemic. According to the national plan, all asymptomatic patients with exposure or suspected exposure to confirmed infection were quarantined, besides residents and nationals returning back to Qatar from other countries. Quarantine is known to have negative psychological effects, including post-traumatic stress symptoms, confusion and irritation. This is amplified by stressors, such as longer quarantine duration, fears of infection, frustration, boredom, inadequate supplies, inadequate information, financial loss and stigma. ${ }^{17}$ From an operational perspective, quarantine sites were set up as extensions of the current government healthcare facilities and were required to monitor both physical and mental health issues. Multidisciplinary teams were set up for specialised medical attention (medical, mental health and psychosocial) to deal with any health crisis that might affect the quarantined individuals. Besides phone consultations, a virtual medical software was installed to facilitate remote consultations for COVID-19 and non-COVID19-related conditions including psychiatric consultations. The launch of a nationwide public health strategy for quarantine resulted in the commission of 51 national quarantine sites across the country between 26 February 2020 and to date, serving a total number of 25330 people with suspected infection or having contact with a confirmed case.

\section{Psychological support clinics for frontline healthcare staff}

Frontline healthcare workers are particularly susceptible to poor mental health outcomes and report more symptoms of anxiety and depression. They feel overwhelmed because of the fear of being infected and spreading the disease to their family and friends. In the current pandemic, similar concerns have been reported from the frontline healthcare workers in Qatar who are directly involved in the diagnosis, treatment and care of patients with COVID-19. They are at risk of poor mental well-being and psychological distress. Mental health services have responded to this challenge by introducing staff support clinics in COVID-19 designated hospitals. Healthcare workers also have access to a confidential helpline.

\section{Public health messaging}

In Qatar, the media is considered as a primary source of information about mental health issues and, therefore, the information provided 
by the media is central to shaping the public's knowledge and attitude. ${ }^{12}$ $\mathrm{MoPH}$ took proactive measures during this pandemic to focus public health messaging on mental health as well. Information management is key during pandemics as limited or incorrect information is known to contribute to anxiety and fear. ${ }^{18}$ Mental health services have actively contributed to content delivered by $\mathrm{MoPH}$ through local TV, social media and newspapers. More than 600000 text messages have been directed to manual and craft workers through the main telecom providers. The messages focus on measures to minimise the psychological consequences associated with restrictions and isolation, also to optimize mental well-being.

\section{Research}

Research funding has been made available to study the psychological impact of COVID-19 to inform evidence-based culturally appropriate interventions and resource allocations. Researchers must prioritise and coordinate essential mental health research during this pandemic, act rapidly and collaboratively to deal with the growing threats to mental health as the epidemic unfolds. The case for integrating mental and physical health research is more pressing than ever. It is paramount to establish and predict the mental health needs by collecting high-quality data on the mental health effects of the COVID-19 pandemic.

\section{CONCLUSION AND FUTURE PRIORITIES}

The necessity of maintaining a functioning mental health service in the context of this pandemic was recognised by the State of Qatar. The mental health services developed successful innovative strategies to deal with the pandemic and its effects on mental health. The strategy to manage COVID-19 patients in seperate designated facilities, adapting a hybrid model with the use of digital health technology and the introduction of helplines have been important changes to increase access to our services. We recognise that the utility of telepsychiatry for future nonpandemic times can provide more accessible services that are culturally more acceptable. ${ }^{19}$ Finally, as the pandemic continues to develop, it is paramount to plan for long-term sustainability from the outset and strengthen the mental health system as a whole.

Acknowledgements We thank Mr. lain Francis Tulley, Chief Executive Mental Health Service, Hamad Medical Corporation and National Lead for Mental Health, State of Qatar, for his contribution in the preparation of this manuscript.

Contributors All authors contributed to the planning, conduct and reporting of the work described in the article. Each author contributed to the subsections of the article. OW: introduction, mental health services in the state of Qatar and challenges; SR: changes to inpatient hospital and acute care strategy to minimise spread of infection in main psychiatry hospital; $\mathrm{JL}$ : psychiatric beds in COVID-19 designated hospitals and consultation-liaison services in general hospitals; OW: modifications to ambulatory and community care; RASA: national mental health helpline; NAM: psychological support for people in isolation and quarantine centres; JL: psychological support clinics for frontline healthcare staff: MAYAA-A: public health messaging; research; conclusion; and future priorities. All the authors read and approved the final manuscript. MAYAA-A is responsible for the overall content as guarantor.

Funding The authors have not declared a specific grant for this research from any funding agency in the public, commercial or not-for-profit sectors.

Competing interests None declared.

Patient consent for publication Not required.

Provenance and peer review Not commissioned; externally peer reviewed.

\section{(2) OPEN ACCESS}

Open access This is an open access article distributed in accordance with the Creative Commons Attribution Non Commercial (CC BY-NC 4.0) license, which permits others to distribute, remix, adapt, build upon this work non-commercially, and license their derivative works on different terms, provided the original work is properly cited, appropriate credit is given, any changes made indicated, and the use is non-commercial. See: http://creativecommons.org/licenses/by-nc/4.0/.

(C) Author(s) (or their employer(s)) 2020. Re-use permitted under CC BY-NC. No commercial re-use. See rights and permissions. Published by BMJ.

\section{Check for updates}

To cite Wadoo 0, Latoo J, Reagu SM, et al. General Psychiatry Epub ahead of print: [please include Day Month Year]. doi:10.1136/gpsych-2020-100313
Received 15 June 2020

Revised 30 July 2020

Accepted 27 August 2020

General Psychiatry 2020;33:e100313

doi:10.1136/gpsych-2020-100313

ORCID iD

Ovais Wadoo http://orcid.org/0000-0001-5023-9245

\section{REFERENCES}

1 Adhanom Ghebreyesus T. Addressing mental health needs: an integral part of COVID-19 response. World Psychiatry 2020;19:129-30.

2 Planning and Statistics Authority. State of Qatar: planning and statistics authority; C, 2020. Available: https://www.psa.gov.qa/ en/Pages/default.aspx [Accessed 1 Jun 2020].

3 Qatar National health strategy 2018-2022 General Secretariat of the Supreme Council of health 2018.

4 Saeed E, Wadoo O, Ouanes S. Community Mental Health services. In: Haque A Gilstrap LL, Ghuloum S, eds. Mental health in Qatar: challenges and prospects. Cambridge Scholars Publishing, 2020: 364-86.

5 Ministry of Public Health. State of Qatar: Ministry of public health; C, 2020. Available: https://covid19.moph.gov.qa/EN/Pages/ default.aspx [Accessed 9 Jun 2020].

6 Abu-Raddad LJ, Chemaitelly H, Ayoub HH, et al. Characterizing the Qatar advancedphase SARS-CoV-2 epidemic. medRxiv 2020.

7 Mazza C, Ricci E, Biondi S, et al. A nationwide survey of psychological distress among Italian people during the COVID-19 pandemic: immediate psychological responses and associated factors. Int J Environ Res Public Health 2020;17:3165.

8 Qiu J, Shen B, Zhao M, et al. A nationwide survey of psychological distress among Chinese people in the COVID-19 epidemic: implications and policy recommendations [published correction appears in Gen Psychiatr. Gen Psychiatr 2020;33:e100213.

9 Fukuta Y, Muder RR. Infections in psychiatric facilities, with an emphasis on outbreaks. Infect Control Hosp Epidemiol 2013;34:80-8.

10 Li S, Zhang Y. Mental healthcare for psychiatric inpatients during the COVID-19 epidemic. Gen Psychiatr 2020;33:e100216.

11 Chevance A, Gourion D, Hoertel N, et al. Ensuring mental health care during the SARS-CoV-2 epidemic in France: a narrative review. Encephale 2020:30073-7.

12 Elzamzamy K, Alsiddiqi A, Khalil A, et al. Newspaper depiction of mental and physical health in Qatar. BJPsych International 2020;23:1-4.

13 Elzamzamy K, Wadoo O. Media and Mental Health. In: Haque A, Gilstrap LL, Ghuloum S, eds. Mental health in Qatar: challenges and prospects. Cambridge Scholars Publishing, 2020: 314-34.

14 Ghuloum S, Bener A, Abou-Saleh MT. Prevalence of mental disorders in adult population attending primary health care setting in Qatari population. J Pak Med Assoc 2011;61:216-21.

15 D'Agostino A, Demartini B, Cavallotti S, et al. Mental health services in Italy during 
the COVID-19 outbreak. Lancet Psychiatry 2020;7:385-7.

16 Siskind D, Honer WG, Clark S, et al. Consensus statement on the use of clozapine during the COVID-19 pandemic. $J$ Psychiatry Neurosci 2020;45, :222-3.

17 Brooks SK, Webster RK, Smith LE, et al. The psychological impact of quarantine and how to reduce it: rapid review of the evidence. Lancet 2020;395:912-20.

18 Shigemura J, Ursano RJ, Morganstein $\mathrm{JC}$, et al. Public responses to the novel 2019 coronavirus (2019-nCoV) in Japan: mental health consequences and target populations. Psychiatry Clin Neurosci 2020;74:281-2.
19 Karim MA, Wadoo O, Reagu SM, et al. Telepsychiatry in the Arabian Gulf region - Implications beyond the Covid-19 pandemic. Asian J Psychiatr 2020;54:102397.

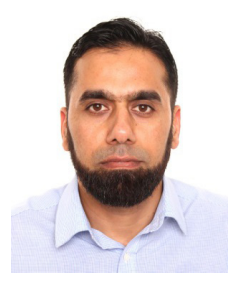

Dr. Wadoo completed his basic training in psychiatry in the UK in 2008, obtaining membership of the Royal College of Psychiatrists (MRCPsych). In 2011, he completed his higher specialist training and was registered as a specialist in general adult psychiatry and rehabilitation psychiatry with the General Medical Council, UK. In the same year, he obtained his master's degree in healthcare management, with distinction, from the Liverpool John Moores University. He is currently a member of the British Neurosciences Association (UK), Royal College of Psychiatrists (UK), International Brain Research Organization (France) and Federation of European Neuroscience Societies (Germany). He has worked as a Consultant Psychiatrist in the National Health Service in England before joining Hamad Medical Corporation in 2017. In addition, he is also working as a Senior Consultant in the Community Mental Health Services in Doha. He is the Director of the Fellowship Programme and Chair of the CPG committee of the Mental Health Services. His main research interests include clinical service, education, various service development and quality initiatives within mental health services. 